\title{
Isotope Effect on the Paramagnetic Resonance of Triplet Excitons*
}

\author{
Trent D. Buckman, O. Hayes Griffith, $\dagger$ and \\ HaRdeN M. MCCONNELL \\ Stauffer Laboratory of Physical Chemistry, Stanford, California
}

(Received 25 June 1965)

$\mathbf{I}^{\mathrm{N}}$ $\mathrm{N}$ previous work ${ }^{1}$ the rate of triplet-exciton jumping in ionic molecular crystals has been deduced under the assumption that nuclear hyperfine interactions make a substantial or dominant contribution to the observed (very narrow) exciton-resonance linewidths. In the present work the magnetic resonance of excitons in (morpholinium) ${ }^{+}$(perdeutero TCNQ) ${ }^{-}$and in $\left(\phi_{3} \mathrm{AsCH}_{3}\right)^{+}$(perdeutero TCNQ $)_{2}{ }^{-}$have been observed, and do, in fact, support the above assumption.

Perdeutero TCNQ was prepared by exchanging 1,4-bis-(dicyanomethylene)-cyclohexane (I) with $\mathrm{D}_{2} \mathrm{O}$ dimethoxyethane mixtures at reflux under an atmosphere of dry nitrogen for $24 \mathrm{~h}$. Three such exchanges gave I containing 82 at. \% deuterium. Dehydrogenation of $\mathrm{I}$ in $\mathrm{CD}_{3} \mathrm{CN}$ gave TCNQ containing 92 at. \% deuterium. Crystals were grown according to the procedure of Acker and Hertler, ${ }^{2}$ and were used immediately or were stored at $77^{\circ} \mathrm{K}$ to prevent the otherwise substantial time-dependent exciton-resonance line broadening.

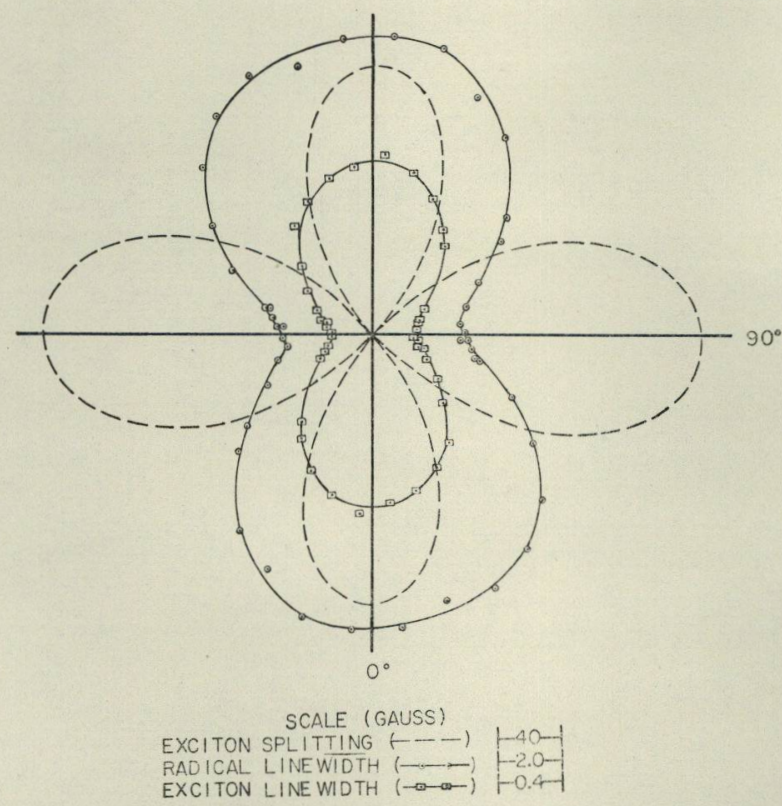

FIG. 1. The exciton linewidth, free-radical impurity linewidth, and exciton fine-structure splitting as a function of angle with the magnetic field in the (001) plane of a (morpholinium) + (perdeutero TCNQ) ${ }^{-}$crystal. At $90^{\circ}$ the magnetic field is parallel to the (110) axis. The linewidths are measured from positions of maximum slope of the $85^{\circ} \mathrm{K}$ ESR absorption curve.
Figure 1 shows the triplet exciton and impurity linewidths in the morpholinium salt as the applied field is rotated in the $(001)^{3}$ plane. It is noted that there is a marked similarity between the exciton linewidth anisotropy and the free-radical linewidth anisotropy in this plane. This suggests that the free-radical impurity is $\mathrm{TCNQ}^{-}$, that this radical has an orientation similar to that of the (TCNQ) ${ }_{2}-$ pairs on which the triplet excitons move, and that the exciton linewidth anisotropy does indeed arise from nuclear hyperfine interactions. ${ }^{1}$ The exciton linewidth anisotropy is evidently not due to the combined effects of exciton fine-structure splittings and crystal imperfections, since the exciton linewidth is not maximal where the fine-structure splitting is most strongly dependent on crystal orientation.

The linewidth of the exciton resonance in the morpholinium salt with protonated TCNQ is approximately $20 \%$ greater than in the corresponding deuterated salt when the magnetic field is in the (001) plane and perpendicular to the (110) direction. The two linewidths are essentially the same when the applied field is in the (001) plane and perpendicular to $(1 \overline{1} 0) .^{4}$ Quite similar results were obtained with the arsonium compound at $85^{\circ} \mathrm{K}$, except that the maximum isotope effect was found to be one and a half times larger than that observed for the morpholinium salt. ${ }^{5}$

* Supported in part by NSF Grant GP 3430, and in part by AEC Contract AT (04-3)-326.

$\dagger$ NAS-NRC Postdoctoral Fellow.

${ }^{1}$ D. D. Thomas, A. W. Merkl, A. F. Hildebrandt, and H. M. McConnell, J. Chem. Phys. 40, 2588 (1964).

${ }^{2}$ D. S. Acker and W. R. Hertler, J. Am. Chem. Soc. 84, 3370 (1962).

${ }^{3}$ M. Maréchal and H. M. McConnell, J. Chem. Phys. (to be published).

${ }^{4}$ The protonated and deuterated TCNQ salts used in these experiments were prepared under as similar conditions as possible to maintain approximately the same level of free-radical impurity. This is necessary because the exciton linewidths may be broadened by radical-exciton exchange interactions. Defining the radical concentration as in Ref. 1 , the radical concentration in the deuterated salt was found to be equal to or slightly greater than in the corresponding protonated salt.

${ }^{5}$ The measurements on the $\left(\phi_{3} \mathrm{AsCH}_{3}\right)^{+}(\mathrm{TCNQ})_{2}^{-}$crystals were complicated at certain orientations by extraneous linebroadening effects. These apparently were caused by crystal imperfections and were not reproducible from crystal to crystal. The minimum linewidths observed were reproducible and were evidently not affected by this problem. These minimum linewidths were used to determine the extent of the isotope effect. Exclusion of oxygen during crystal preparation had no apparent effect on the exciton linewidths. 
